\title{
lowa: Landluft macht Atemwege nicht frei
}

Vom Aufwind in den Gegenwind - die Hygienehypothese erfährt zur Zeit nicht nur Bestätigung. So stellen jetzt die Ergebnisse einer bevölkerungsbasierten US-Studie die Atopie-Schutzwirkung eines Aufwachsens in ländlicher Umgebung in Frage.

\begin{abstract}
E pidemiologen schrieben alle FamiC lien mit Kindern zwischen 6 und 14 Jahren an, die in zehn Schulregistern von zwei nicht benachbarten, aber die gleichen Produkte herstellenden ländlichen Bezirken des US-Bundesstaates Iowa aufgeführt waren. Den Schreiben waren Fragebögen beigefügt, die demographische Faktoren und mögliche Asthmasymptome erheben sollten. Dabei kam u. a. der ISAAC Core Questionnaire zum Einsatz, ein international anerkanntes Instrument zur Erfassung der Wheezing- und Asthmaprävalenz.

Die Rücklaufquote war mit 86,6\% sehr hoch. Die 12-Monats-Prävalenz des Symptoms Wheezing betrug 19,1\%, über eine ärztliche Asthmadia-
\end{abstract}

gnose berichteten $13,4 \%$ der Angeschriebenen. In der Multivarianzanalyse ergab sich für Kinder vom Land im Vergleich zu Kindern in der Stadt ein geringeres Risiko für Wheezing, sowohl bezogen auf die Gesamtlebenszeit (Odds Ratio 0,71) als auch auf das letzte Jahr (Odds Ratio 0,77). Allerdings waren diese Zusammenhänge nur in einem der beiden Bezirke signifikant. Wurde das Symptom Wheezing angegeben, war sowohl auf dem Land wie auch in der Stadt die ärztliche Diagnose Asthma in vergleichbarer Häufigkeit zu finden, auch die Morbidität war vergleichbar.

Viel bemerkenswerter ist allerdings, dass sich die ermittelte Gesamtrate von Wheezing bzw. Asthma nur wenig von den Werten aus Großstädten
Eine Kindheit im

Schatten des Sternenbanners

schützt nicht besonders gut vor Asthma.

wie Chicago (Wheezing-Prävalenz 19,8\%) oder Seattle (Wheezing-Prävalenz 22,9\%) unterschied. Im Vergleich zu ländlichen Gebieten Europas sind die erhobenen Prävalenzzahlen dagegen erstaunlich hoch. Dies kann studientechnische Gründe haben oder auch auf unterschiedliche agrartechnische Produktionsweisen hindeuten.

Fazit: Die Asthma- und Wheezing-Prävalenz in ländlichen Bezirken im USamerikanischen Mittelwesten ist kaum geringer als in amerikanischen Großstädten, allerdings sehr viel höher als in ländlichen Bezirken Europas.

$b k$

Chrischilles E et al. Asthma prevalence and morbidity among rural lowa schoolchildren. J Allergy Clin Immunol 2004; 113: 66-71

\section{Erhöhtes Rhinitisrisiko durch Antibiotika?}

\section{Manche durchgemachten Infektionen korrelieren invers mit dem Atopierisiko. Hierfür gibt es zahlreiche Belege. Nicht ganz so klar ist die Beziehung zwischen der Einnahme von Antibiotika in den ersten Lebensjahren und dem Auftreten einer Atopie.}

$M$ it Hilfe zweier großer Fall-Kontrollstudien - eine mit 40.183 Kindern aus 141 Praxen und eine mit 76.310 Kindern aus 464 Praxen - versuchten britische Forscher einem $\mathrm{Zu}$ sammenhang zwischen Antibiotikagabe und einem späteren Heuschnupfen auf die Spur zu kommen. Alle Kinder wurden während ihres 1 . Lebensjahres in die Untersuchung aufgenommen und über mindestens 5 Jahre beobachtet. 7.098 der Kinder entwickelten im Alter über 2 Jahren eine allergische Rhinitis. Ihnen wurde jeweils ein passendes gesundes Kontrollkind gegenübergestellt.

In der gepoolten Analyse ergab sich zunächst eine Odds Ratio für Heu- schnupfen von 1,11 bei Einnahme eines Antibiotikums im 1. Lebensjahr, von 1,35 bei Einnahme im 2. Lebensjahr und von 1,47 bei Einnahme im 3. Lebensjahr. Wurden die Berechnungen allerdings um den Faktor „Häufigkeit von Arztbesuchen" bereinigt, verschwand das Risiko, er ergaben sich Odds Ratios von 0,92, 1,05 bzw. 1,10.

Aufgeschlüsselt nach Antibiotikaklassen war zunächst eine deutliche Korrelation zwischen den am häufigsten verschriebenen Penizillinen und Makroliden und dem Heuschnupfenrisiko zu erkennen. Nach Adjustierung hinsichtlich der Anzahl der Arztbesuche verschwand aber auch dieser Zusammenhang. Keine
Korrelation errechnete sich bei den Cephalosporinen, die ein besonders breites antibakterielles Wirkspektrum haben.

Frühere Studien, die einen $\mathrm{Zu}$ sammenhang zwischen Antibiotikaeinnahme und Atopie ergeben hatten, waren möglicherweise mit einem systematischen Fehler behaftet oder hatten die Tendenz mancher Familien nicht berücksichtigt, mit ihren Kindern häufiger als andere einen Arzt aufzusuchen.

Fazit: Die Hypothese, wonach der Einsatz von Antibiotika vor allem im 1. Lebensjahr das Atopierisiko erhöht, bestätigte sich in dieser Studie nicht. Auch von Breitspektrumantibiotika geht offenbar keine besondere Gefahr aus. $\quad b k$

Bremner SA et al. Early-life exposure to antibacterials and the subsequent development of hayfever in childhood in the UK: case-control studies using the General Practice Research Database and the Doctors' Independent Network. Clin Exp Allergy 2003; 33: 1518-25 